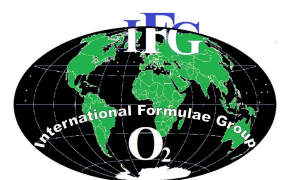

ISSN 1997-342X (Online), ISSN 1991-8631 (Print)

\title{
Etude pédologique et phytosociologique de deux mares dans le Parc National du Niokolo Koba dans un contexte d'invasion biologique par Mimosa pigra
}

\author{
Mallé GUEYE ${ }^{1 *}$ et Kandioura NOBA ${ }^{2}$ \\ ${ }^{1}$ Parc National du Niokolo Koba, BP 37, Tambacounda, Sénégal. \\ ${ }^{2}$ Laboratoire de Botanique et Biodiversité, Département de Biologie Végétale, Faculté des Sciences et \\ Techniques, Université Cheikh Anta Diop de Dakar, BP 5005, Dakar-Fann, Sénégal. \\ *Auteur correspondant; E-mail : mallegueye@yahoo.fr, Tél. (221) 775682526.
}

\section{RESUME}

Dans le Parc National du Niokolo Koba, l'invasion biologique des mares par Mimosa pigra a pris des proportions inquiétantes depuis quelques années. L'objectif de cette étude est de comparer les mares de Nianaka et Dalafourounté afin d'identifier les facteurs qui favoriseraient l'invasion de cette espèce. Pour ce faire, nous avons réalisé des études pédologiques et phytosociologiques à l'intérieur, en bordure et au pourtour de ces mares. Les résultats obtenus ont révélé que Mimosa pigra se developpe sur un sol argileux lourd à nutrition minérale correcte chez les deux mares. De plus, l'analyse de la flore révèle que 35 espèces appartenant à 26 genres répartis en 16 familles ont été recensées à Dalafourounté contre 24 appartenant à 20 genres répartis dans 13 familles à Nianaka. Chez cette dernière, la proportion de dicotylédones $(83,3 \%)$ est inférieure à celle de Dalafourounté $(91,4 \%)$ et vice versa pour les monocotylédones $16,7 \%$ contre $8,6 \%$. En outre, la flore des deux mares présente une dominance des Combretaceae et des Mimosaceae. Par ailleurs, Mimosa pigra est invasive exclusivement à Nianaka et se comporte de manière constante en bordure et à l'intérieur de la mare alors qu'elle est fréquente autour de celle-ci. Au terme de cette étude, l'invasion de Mimosa pigra serait due au relief et au niveau de remplissage des mares.

() 2015 International Formulae Group. All rights reserved.

Mots clés : Flore, sol, invasion, Nianaka, Dalafourounté.

\section{Soil and phytosociological study of two bonds in the Niokolo Koba National Park in a context of biological invasion by Mimosa pigra}

\begin{abstract}
The biological invasion of ponds by Mimosa pigra has been an increasing threat in Niokolo Koba National Park for the past years. This study aims at comparing the Nianaka and Dalafourounté ponds in order to identify the factors behind the invasion of these species. In this respect, we have conducted pedological and phytosociological studies inside, at the edge and around these ponds. The results of the study have shown that Mimosa pigra, in both ponds, grows on a heavy clayey soil with proper mineral nutrition. Moreover, the flora analysis reveals that 35 species belonging to 26 genera divided into 16 families have been inventoried at
\end{abstract}


Dalafourounté while 24 species within 20 genera divided into 13 families have been identified at Nianaka. The proportion of dicotyledons is lower at Nianaka (83.3\%) than at Dalafourounte $(91.4 \%)$ and the opposite is noticed with monocotyledons, $16.7 \%$ against $8.6 \%$. Besides, there is a dominance of Combretaceae and Mimosaceae in the flora of both ponds. In addition, Mimosa pigra is only invasive at Nianaka where it is permanently present at the edge and inside the pond while its presence around it is not constant. As a final result of this study, the Mimosa pigra invasion could be originating from the relief and the pond filling up.

(C) 2015 International Formulae Group. All rights reserved.

Keywords: Flora, soil, invasion, Nianaka, Dalafourounté.

\section{INTRODUCTION}

Les espèces exotiques invasives sont des espèces qui s'établissent et répandent leurs menaces sur des écosystèmes et des habitats variés. Ce sont des espèces à nuisance économique et environnementale (McNeely et al., 2001). C'est le cas de l'amourette (Giant sensitive en anglais), Mimosa pigra qui est native d'Amérique Centrale (Mexique) et du Nord de l'Amérique du Sud. Elle a été introduite en Afrique, en Asie du Sud-Est, sur certaines îles du Pacifiques et en Australie vraisemblablement à partir de jardins botaniques et/ou en tant que curiosité à cause du mouvement de ses feuilles (Lonsdale et al., 1989 ; Walden et al., 1999). Mimosa pigra est considérée comme l'espèce végétale la plus invasive dans les zones marécageuses tropicales (Cronk et Fuller, 1995).

En Afrique, Mimosa pigra est présente à l'heure actuelle dans 43 pays (Invasive Species Compendium, CABI, 2013) et fait partie des 100 espèces invasives les plus problématiques au monde (Lowe et al., 2000).

Au Sénégal, elle est présente dans les vallées et les dépressions qui restent inondées pendant une bonne partie de l'année ou sur les rivages des cours d'eau (Bâ et al., 2008). Des observations dans le Parc National du Niokolo Koba, ont montré que les dégâts occasionnés par cette espèce sont considérables. Il s'agit de perte de biodiversité et de fermeture de certaines mares, empêchant ainsi ces milieux de remplir leurs fonctions écologiques (Gueye, 2014). Présentement, plusieurs mares du parc sont colonisées par cette espèce végétale invasive avec un degré d'infestation très sévère (Ndiaye, 2000).

C'est dans ce contexte que cette étude a été initiée afin d'identifier aux plans phytosociologique et pédologique les causes, les facteurs qui favorisent l'invasion des mares du Parc National du Niokolo Koba par Mimosa pigra.

\section{MATERIEL ET METHODES \\ Zone d'étude}

Le Parc National du Niokolo Koba est situé au Sud-Est du Sénégal, entre les parallèles $12^{\circ} 30$ et $13^{\circ} 20$ de latitude nord et entre les méridiens $18^{\circ} 30$ et $13^{\circ} 42$ de longitude ouest (Figure 1). Il couvre une superficie de $9130 \mathrm{~km}^{2}$ selon Renaud et al. (2006). Le relief est constitué de plusieurs unités géomorphologiques telles que des plateaux, des vallées et des collines. Le climat est de type soudano-guinéen influencé par deux masses d'air, les alizés et la mousson, mais également par les précipitations et les températures (DPN, 2000). Il correspond au domaine climatique de type tropical avec saison sèche en hiver (Aw) d'après la classification de Köppen (Gueye, 2014). Les hauteurs de pluies varient entre 900 et 1200 $\mathrm{mm}$ avec une saison pluvieuse allant de juin à octobre alors que les températures oscillent entre $25{ }^{\circ} \mathrm{C}$ en décembre et $33{ }^{\circ} \mathrm{C}$ en mai (Ndiaye, 2012). Le réseau hydrographique du parc est constitué par le fleuve Gambie et ses principaux affluents, le Niokolo Koba et la Koulountou, ainsi que de plusieurs mares et étangs (Ndiaye, 2012). Le parc renferme plus 
de 1500 espèces de plantes à fleurs (Adam, 1971; Schneider \& Sambou., 1982), soit $62 \%$ des espèces de plantes à fleurs du Sénégal.

La phase de terrain de cette étude s'est réalisée en 2011 durant le mois d'avril, plus précisément au niveau des mares de Nianaka (X : $13^{\circ} 02^{\prime} 42^{\prime \prime} \mathrm{N}$; Y : $13^{\circ} 18^{\prime} 50^{\prime}$ 'O) et Dalafourounté $\quad\left(\mathrm{X}: 1^{\circ} 011^{\prime} 46^{\prime}{ }^{\prime} \mathrm{N}\right.$; $\mathrm{Y}$ : $\left.13^{\circ} 14^{\prime} 14^{\prime}, \mathrm{O}\right)$.

\section{Matériel}

Le matériel utilisé pour l'étude phytosociologique des zones d'infestation de Mimosa pigra est constitué de manuels tels que Flore du Sénégal (Berhaut, 1967), Nouvelle Flore illustrée du Sénégal et des régions voisines (Berhaut, 2011), Arbres, Arbustes et Lianes des Zones Sèches d'Afrique de l'Ouest (Arbonnier, 2010). Un GPS (Global Positioning System) de marque GARMIN etrex et un appareil photo numérique NIKON ont été utilisés respectivement pour le géo-référencement et la prise d'images au niveau des sites. De plus, une pioche, une pelle de terrain et des sachets pour contenir les échantillons de sols ont été également utilisés.

\section{Méthodologie \\ Etude pédologique}

En ce qui concerne la caractérisation des sols, la méthodologie a consisté à faire des prélèvements d'échantillons de sols qui par la suite ont été analysés au niveau du Laboratoire des Moyens Analytiques (LAMA certifié ISO 9001: 2000) de l'Institut de Recherche pour le Développement (IRD) de Dakar afin de connaître les paramètres qui interviennent dans le processus d'invasion de Mimosa pigra dans le Parc. En plus des deux mares étudiées (Nianaka et Dalafourounté), des échantillons de sols en provenance d'autres sites ont été analysées pour aider à la compréhension du problème. Il s'agit de Simenti, Kountadala tous les deux situé dans le parc. Le choix de la mare de Dalafourouté se justifie par le fait qu'à l'état actuel, aucun spécimen de Mimosa pigra n'y a été observé. Les différentes caractéristiques déterminées sont: la granulométrie, le $\mathrm{pH}$, la teneur en azote total, en carbone total, en phosphore total et assimilable, les bases échangeables et la capacité d'échange.

\section{Etude phytosociologique}

Ensuite, il s'est agi de faire une étude phytosociologique au niveau des mares (autour, bordure et intérieur) de Nianaka et de Dalafourounté. Elle a été effectuée au niveau de ces mares dans des stations de relevé qui sont, selon Godron (1968), des espaces à végétation uniforme dans lesquels les conditions écologiques sont homogènes. La méthode de Vanden (1982) a été adoptée pour faire l'inventaire floristique. Les plantes non identifiés ont été récoltées, codées et gardées sous presse spécimen pour être identifiées ultérieurement au Laboratoire de Botanique de l'Université de Dakar. Après un relevé global de la zone, il a fallu faire une estimation générale du recouvrement de chaque espèce. La méthode de Braun-Blanquet (1932) a été adoptée à cet effet.

\section{Traitement et analyse des données}

Les données pédologiques ont été traitées puis analysées sur la base de la classification texturale de Jamagne (1967) afin de déterminer la catégorie de sols pour chaque site étudié.

En ce qui concerne les données phytosociologiques, elles ont fait l'objet d'une analyse quantitative de la structure de la végétation réalisée à l'aide de la méthode de Caratini (1985). L'indice d'abondance/dominance moyen (Adm) est calculé par rapport au nombre de relevés dans lesquels l'espèce est présente. Pour le calcul de cet indice, les codes semi-quantitatifs d'abondance/dominance sont rendus quantitatifs (Gillet, 2000). L'échelle de BraunBlanquet (1932) n'étant pas linéaire, il est préférable de convertir chaque code en un recouvrement moyen, à l'aide de la table de 
correspondance entre le code d'abondancedominance (AD code), l'indice quantitatif d'abondance-dominance (AD num) et le recouvrement moyen, minimum et maximum.

\section{RESULTATS}

\section{Caractéristiques des sols}

Les résultats des analyses pédologiques sont consignés dans les Tableaux 1 et 2 . Ils révèlent que les populations de Mimosa pigra sont observées sur des sols de textures relativement différentes (Tableau 1). Nianaka, Dalafourounté et Kountadala présentent un sol à texture argileuse lourde alors que Simenti affiche un sol argileux. Parallèlement, les résultats de l'analyse chimique révèlent que l'ensemble des sites enregistrent un sol ayant des $\mathrm{pH}\left(\mathrm{pH}_{\mathrm{H} 2 \mathrm{O}}\right.$ et $\left.\mathrm{pH}_{\mathrm{KCl}}\right)$ inférieur à 5. De plus, la différence entre ces $\mathrm{pH}$ est positive pour tous les sites de prélèvement. En outre, Nianaka présente les taux d'azote les plus élevées suivi respectivement par Dalafourounté, Simenti et Kountadala. Toutefois, le rapport carbone total sur azote total est égal à 11 pour l'ensemble des sites à l'exception de Nianaka qui donne la valeur de 10. Simenti enregistre plus de phosphore total et la plus petite valeur pour le phosphore assimilable. Le site de Dalafourouté, quant à lui, affiche la deuxième meilleure valeur en phosphore (total et assimilable) et au dessus des autres sites pour le phosphore assimilable. Par ailleurs, les résultats obtenus sur bases échangeables révèlent que Nianaka donne les valeurs les plus élevées (Tableau 2). En ce qui concerne la capacité d'échange cationique, les résultats montrent que le site de Nianaka enregistre la valeur la plus grande suivi de Dalafourounté. Les résultats de l'analyse pédologique montrent que le rapport carbone total sur azote total donne une valeur égale à 11 pour l'ensemble des sites à l'exception de Nianaka qui affiche un rapport égal à 10 (Tableau 3). Les rapports $\mathrm{Ca} / \mathrm{K}, \mathrm{Ca} / \mathrm{Mg}$ enregistrent respectivement une valeur moyenne de 9,6 et 1,4 alors que $\mathrm{Mg} / \mathrm{K}$ et
$(\mathrm{Ca}+\mathrm{Mg}) / \mathrm{K}$ donnent respectivement 6,8 et 16,4 .

\section{Etude de la flore}

\section{Analyse qualitative de la flore}

Le spectre taxonomique de la flore des mares de Nianaka et de Dalafourounté est présenté dans le Tableau 4 en fonction des familles, genres et espèces. Il ressort que la flore autour des mares étudiées est riche de 20 et 33 espèces végétales, respectivement à Nianaka et Dalafourounté, réparties dans 10 et 14 familles et 16 et 24 genres (Tableau 4). La richesse spécifique est 5 et 4 respectivement pour l'intérieur et la bordure de la mare de Nianaka. Le même décompte a été enregistré pour ce qui est des genres et des familles pour ce site. Parallèlement, Dalafourounté affiche un inventaire identique en ce qui concerne le nombre d'espèces, le nombre de genres et de familles aussi bien à l'intérieur comme en bordure de la mare. La liste des espèces recensées au niveau de Nianaka et Dalafourounté sont consignées dans le Tableau 5. Les résultats montrent que le nombre total d'espèces végétales inventoriées aussi bien à l'intérieur, en bordure qu'à l'intérieur des mares s'élève à 35 à Dalafourounté et 24 à Nianaka.

Les résultats obtenus sur les structures de la flore des mares de Nianaka et Dalafourounté sont consignés dans le Tableau 6. L'ensemble des relevés réalisés au niveau des mares étudiées a permis de recenser à Nianaka 24 espèces végétales appartenant à 20 genres répartis dans 13 familles. Les Dicotylédones représentent $83,3 \% \quad(20$ espèces) et les Monocotylédones 16,7\% (4 espèces) de cette flore (Tableau 6). Parallèlement, les résultats montrent qu'à Dalafourounté, 35 espèces appartenant à 26 genres répartis en 16 familles ont été recensées. Les Dicotylédones représentent à eux seuls $91,4 \%$ des espèces inventoriées alors que les Monocotylédones enregistrent $8,6 \%$ (Tableau 6). 
Les résultats obtenus à la mare de Nianaka sur l'importance relative des différentes familles botaniques montrent que trois familles regroupent plus de la moitié des espèces recensées : les Combretaceae (25\%), les Caesalpiniaceae $(12,5 \%)$ et les Mimosaceae (16,7\%) (Tableau 7). Les 9 autres familles ayant chacune une espèce représentent $37,8 \%$ des espèces. Il s'agit des Anacardiaceae, des Annonaceae, des Arecaceae, des Borraginaceae, des Cyperaceae, des Cyperaceae, des Fabaceae, des Rhamnaceae, des Rubiaceae et des Sphenocleaceae (Tableau 7). Enfin, la famille des Poaceae totalise $8,3 \%$ des espèces et est représentée par deux espèces.

$\mathrm{Au}$ niveau de la mare de Dalafourounté, les résultats obtenus montrent que la famille des Combretaceae regroupe le quart de des espèces recensées avec $25,7 \%$. Cinq (05) familles botaniques totalisent sensiblement $45,8 \%$ des espèces avec au moins deux espèces dans chacune des familles. Il s'agit des Anacardiaceae, des Caesalpiniaceae, des Mimosaceae, des Poaceae et des Rubiaceae. Enfin, dix (10) familles enregistrent $29 \%$ des espèces avec une seule espèce dans chaque famille.

\section{Analyse quantitative de la flore \\ Etendue de la distribution des espèces dans les mares étudiées}

L'analyse de la fréquence des espèces dans l'ensemble des relevés des mares de Nianaka et Dalafourounté a permis de définir l'étendue de leur distribution. Cinq (05) groupes d'espèces ont été distingués dans chaque site selon les indices de fréquence. Les résultats de l'analyse de la fréquence des espèces sont consignés dans le Tableau 8. A Nianaka la part occupée par les espèces constantes (Indice V) est plus élevée en bordure avec $75 \%$ suivie respectivement de l'intérieur et autour de la mare avec 60 et $30 \%$. En revanche, les espèces abondantes (Indice IV) ne sont présentes qu'à l'intérieur de la mare (40\%) uniquement alors que les espèces fréquentes (Indice III, 10\%) et accessoires (II, 25\%) n'existent que autour de la mare. Quant aux espèces rares ou accidentelles (Indice I), elles représentent $35 \%$ des espèces présentent autour de la mare. A Dalafourounté, la proportion d'espèces constantes (Indice V) est plus élevée en bordure $(66,7 \%)$ qu'aux autres parties de la mare (Tableau 8). De plus, les espèces abondantes (IV) ne sont répertoriées autour et à l'intérieur de la mare avec respectivement 3 et $25 \%$ alors que le fréquentes (Indice III) et les rares ou accidentelles (Indice I) ne sont enregistrées qu'autour (12,1\%). A l'intérieur de la mare de Dalafourounté, $50 \%$ des espèces sont accessoires (Indice II) contre 33,3 en bordure et $18,2 \%$ autour de la mare.

Les résultats de la répartition des espèces recensées dans les mares étudiées selon les index de Caratini (1985) sont consignés dans le Tableau 9. L'analyse de ces résultats montre que parmi ces espèces inventoriées, certaines ne se comportent pas de la même manière à Nianaka et à Dalafourounté. C'est ainsi que trois groupes d'espèces sont distingués :

- Groupe 1: Comportant les espèces qui ne changent pas d'indice aussi bien à Nianaka qu'à Dalafourounté. Dans ce groupe sept (07) espèces sont constantes et se rencontrent dans 80 à $100 \%$ des relevés (Indice V): Acacia sieberiana, Combretum glutinosum, Guiera senegalensis, Mitragyna inermis, Piliostigma thonningii et Pterocarpus erinaceus. De plus, deux (02) espèces sont accessoires et sont rencontrées dans 20 à moins de $40 \%$ des relevés (Indice II). Il s'agit de Anogeissus leiocarpus et Ziziphus mucronata. En outre, Combretum micranthum est rare ou accidentelle et se rencontre dans 0 à moins de $20 \%$;

- Groupe 2 : Concerne les espèces qui changent d'indice aussi bien à Nianaka qu'à Dalafourounté. Il s'agit de Borassus aethiopum, Combretum adenogonium, 
Terminalia macroptera, Acacia seyal, Andropogon gayanus, Cassia sieberiana, Dichrostachys cinerea, Hexalobus monopetalus et Lannea microcarpa ;

- Groupe 3 : Renferme les espèces qui sont présentes exclusivement à Nianaka ou à Dalafourounté. Deux (02) espèces sont exclusives à Nianaka : Mimosa pigra (Indice III) et Senna occidentalis (Indice I) et quatorze (14) espèces à Dalafourounté dont trois (03) en Indice II (Bombax costatum, Gardenia ternifolia, Vetiveria nigricana) et onze (11) en indice I (Acacia macrostachya, Combretum lecardii, Combretum paniculatum, Cordyla pinnata, Lannea velutina, Lannea acida, Sarcocephalus latifolius, Parkia biglobosa, Sterculia setigera, Tapinanthus bangwensis et Baissea multiflora).

Les résultats obtenus sur la répartition des espèces en bordure et à l'intérieur des mares selon les index de Caratini sont dans le Tableau 10. En bordure des mares, ces résultats montrent que certaines espèces se comportent de la même manière aussi bien à Nianaka comme à Dalafourounté. Il s'agit de Mitragyna inermis et Vetiveria nigricana qui sont constantes dans les deux sites et se rencontrent dans 80 à $100 \%$ des relevés (indice V). De plus, Borassus aethiopum change d'indice aussi bien à Nianaka (Indice I) qu'à Dalafourounté (Indice II). Enfin, l'espèce Mimosa pigra est exclusive à Nianaka et se comporte de manière constante.

L'analyse du Tableau 10 montre que les espèces recensées à l'intérieur des deux mares sont toutes différentes sauf Coldenia procumbens. En effet, cette espèce est présente dans les deux sites mais ne s'y comporte pas de la même manière car étant constante à Nianaka (Indice V) et accessoire à Dalafourounté (Indice II). Toutefois, force est de constater que Mimosa pigra n'est présente que dans le premier site cité précédemment.

\section{Abondance des espèces}

Les résultats des abondances dominances moyennes, des fréquences et des index des espèces recensées autour des mares de Dalafourounté et Nianaka sont consignés dans le Tableau 11.

Il ressort de cette étude quantitative des espèces recensées à Dalafourounté que Pterocarpus erinaceus est l'espèce classée constante qui enregistre l'indice d'abondance dominance moyenne la plus élevée $(2,7)$. Toutes les autres espèces présentent un indice d'abondance dominance moyenne compris entre 0,02 et 2. Par contre, à Nianaka, les résultats révèlent que Guiera senegalensis est la seule espèce constante avec une abondance dominance moyenne élevée et égale à 3,2. Elle est suivie par Mitragyna inermis et Combretum glutinosum avec respectivement 2,3 et 1,9 . Le reste des espèces inventoriées autour de cette mare enregistre une valeur comprise entre 0,02 et 0,8. Mimosa pigra quand à elle présente une abondance dominance moyenne égale à 0,3 .

En outre, le Tableau 12 présente les abondances dominances moyennes, les fréquences et les index de Caratini (1985) des espèces recensées en bordure des mares de Dalafourounté et Nianaka. Les résultats de l'analyse quantitaive de la flore présente en bordure des mares étudiées montrent que Vetiveria nigricana est la seule espèce constante présentant une abondance dominance moyenne élevée de 5. Parallèlement, à Nianaka, Vetiveria nigricana, Mimosa pigra et Mitragyna inermis sont constantes et montrent une abondance dominance moyenne élevée et égale à 4, 2,6 et 2 respectivement. De plus, les résulats obtenus révèlent que Vetiveria nigricana est la seule espèce, parmi celles recensées à l'intérieur de la mare de Dalafourounté, qui est constante avec une abondance dominance moyenne élevée et égale à 3,5 (Tableau 13). Par contre, à Nianaka, Mimosa pigra est la seule espèce constante présentant une abondance dominance moyenne élevée de 5 . 


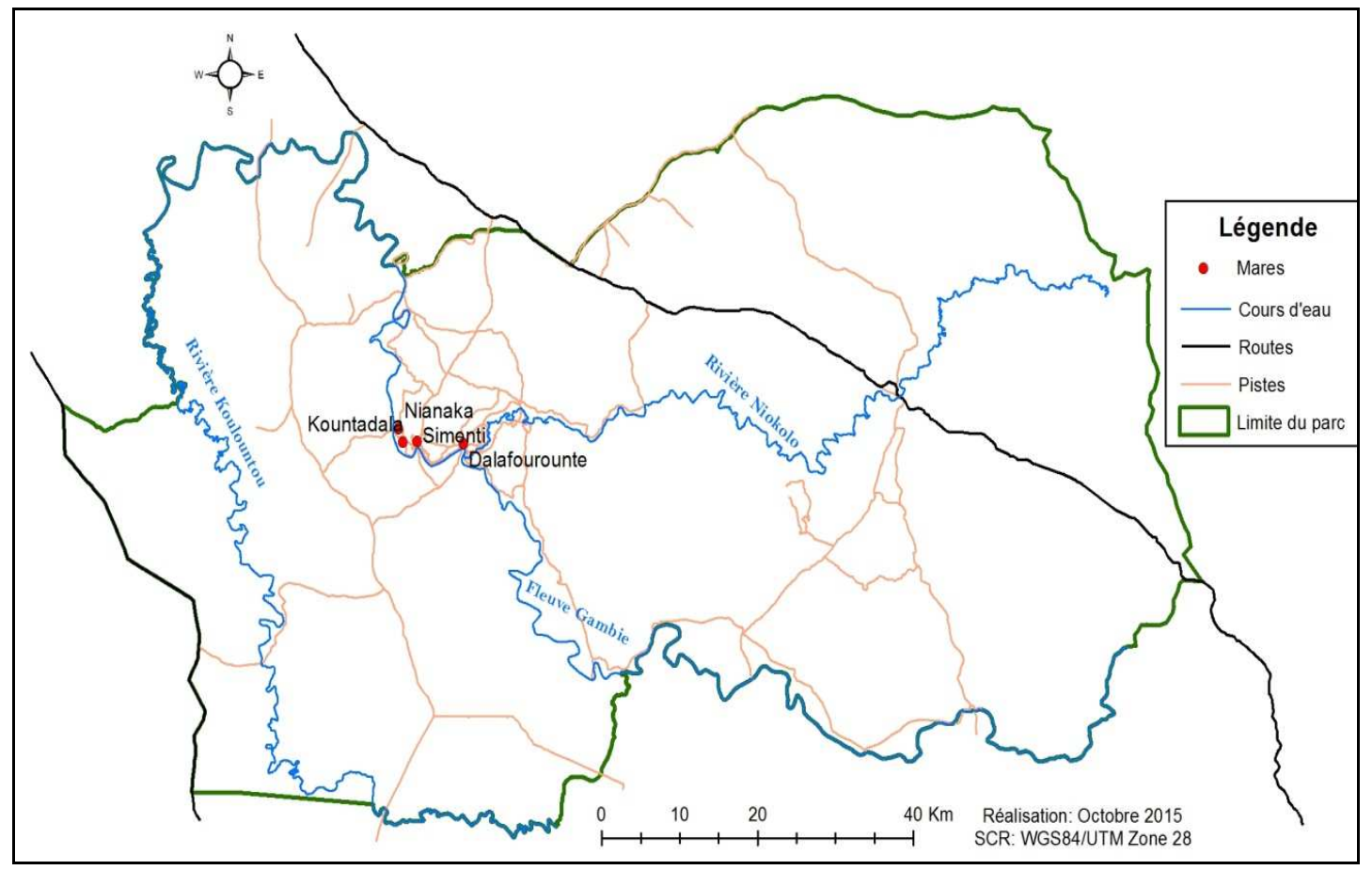

Figure 1 : Carte de la zone d'étude.

Tableau 1: Résultats des analyses de sols sur la granulométrie, pH et macroéléments.

\begin{tabular}{|c|c|c|c|c|c|c|c|c|c|c|c|}
\hline Sites & $\begin{array}{c}\text { A } \\
(\%)\end{array}$ & $\begin{array}{l}\text { LT } \\
(\%)\end{array}$ & $\begin{array}{l}\text { ST } \\
(\%)\end{array}$ & CT & $\mathrm{pH}_{\mathrm{H} 2 \mathrm{O}}$ & $\mathbf{p H}_{\mathrm{KCl}}$ & $\Delta \mathbf{p H}$ & $\begin{array}{l}N_{t} \\
(\%)\end{array}$ & $\begin{array}{c}C_{t} \\
(\%)\end{array}$ & $\begin{array}{c}\mathbf{P}_{\mathrm{t}} \\
(\mathbf{P} \\
\mathrm{mg} / \mathrm{kg})\end{array}$ & $\begin{array}{c}\mathbf{P}_{\mathbf{a}} \\
(\mathbf{P} \\
\mathrm{mg} / \mathrm{kg})\end{array}$ \\
\hline Nianaka & 69,10 & 22,10 & 1 & $\mathrm{AL}$ & 4,6 & 3,4 & 1,2 & 0,16 & 1,58 & 221 & 17 \\
\hline Simenti & 38,60 & 26,70 & 28 & $\mathrm{~A}$ & 4,4 & 3,4 & 1 & 0,13 & 1,45 & 462 & 11 \\
\hline Dalafourounté & 57,60 & 29,50 & 5 & $\mathrm{AL}$ & 4,6 & 3,5 & 1,1 & 0,14 & 1,49 & 268 & 26 \\
\hline Kountadala & 64,80 & 20,60 & 6 & $\mathrm{AL}$ & 4,9 & 3,7 & 1,2 & 0,12 & 1,26 & 249 & 12 \\
\hline
\end{tabular}

Tableau 2: Résultats des analyses de sols sur les bases échangeables et sur la capacité d'échange.

\begin{tabular}{|c|c|c|c|c|c|}
\hline \multirow{2}{*}{ Sites } & \multicolumn{4}{|c|}{$\mathbf{B E}$} & \multirow{2}{*}{$\begin{array}{c}\text { CEC } \\
\text { méq\% }\end{array}$} \\
\hline & Ca méq\% & Mg méq\% & Na méq\% & K méq\% & \\
\hline Nianaka & 5,96 & 4,00 & 0,18 & 0,53 & 19,65 \\
\hline Simenti & 2,56 & 1,41 & 0,06 & 0,24 & 13,30 \\
\hline Dalafourounté & 3,12 & 2,33 & 0,16 & 0,51 & 16,35 \\
\hline Kountadala & 3,59 & 2,94 & 0,13 & 0,25 & 14,40 \\
\hline
\end{tabular}

Ca : Calcium ; Mg : Magnésium ; Na: Azote; K: Potassium; BE: Bases échangeables ;

CE : Capacité d'Echange Cationique. 
Tableau 3 : Rapport entre les éléments minéraux.

\begin{tabular}{lccccc}
\hline \multicolumn{1}{c}{ Sites } & $\mathbf{C} / \mathbf{N}$ & $\mathbf{C a} / \mathbf{K}$ & $\mathbf{C a} / \mathbf{M g}$ & $\mathbf{M g} / \mathbf{K}$ & $(\mathbf{C a}+\mathbf{M g}) / \mathbf{K}$ \\
\hline Nianaka & 10 & 11 & 1 & 8 & 19 \\
Simenti & 11 & 11 & 2 & 6 & 17 \\
Dalafourounté & 11 & 6 & 1 & 5 & 11 \\
Kountadala & 11 & 14 & 1 & 12 & 26 \\
\hline
\end{tabular}

Tableau 4 : Répartition par familles, genres et espèces.

\begin{tabular}{lcccccccccc}
\hline \multirow{2}{*}{ Sites } & \multicolumn{3}{c}{ Familles } & \multicolumn{3}{c}{ Genres } & \multicolumn{3}{c}{ Espèces } \\
\cline { 2 - 11 } & Autour & Intérieur & Bordure & Autour & Intérieur & Bordure & Autour & Intérieur & Bordure \\
\hline Nianaka & 10 & 5 & 4 & 16 & 5 & 4 & 20 & 5 & 4 \\
Dalafourounté & 14 & 4 & 3 & 24 & 4 & 3 & 33 & 4 & 3 \\
\hline
\end{tabular}

Tableau 5 : Liste de présence des espèces recensées dans les mares étudiées.

\begin{tabular}{llcc}
\hline Familles & Espèces & Nianaka & Dalafourounté \\
\hline Anacardiaceae & Lannea acida A. Rich. & & + \\
Anacardiaceae & Lannea microcarpa Engl. Et K. Krause & + & + \\
Anacardiaceae & Lannea velutina A. Rich. & & + \\
Annonaceae & Hexalobus monopetalus (A. Rich.) Engl. et Diels & & + \\
Apocynaceae & Baissea multiflora & & + \\
Arecaceae & Borassus aethiopum Mart. & + & + \\
Bombacaceae & Bombax costatum Pellegr. et Vuillet & & + \\
Borraginaceae & Coledenia procumbens & + & + \\
Caesalpiniaceae & Senna occidentalisL. & + & \\
Caesalpiniaceae & Cassia sieberiana DC. & + & + \\
Caesalpiniaceae & Cordyla pinnata (Lepr.) Milne-Redh. & & + \\
Caesalpiniaceae & Piliostigma thonningii (Schum.) Milne-Redh. & + & + \\
Combretaceae & Anogeissus leiocarpus (DC.) Guill. et Perr. & + & + \\
Combretaceae & Combretum adenogonium Stend. Ex A. Rich. & + & + \\
Combretaceae & Combretum glutinosum Perrott ex DC. & + & + \\
Combretaceae & Combretum lecardii Engl. et Diels & & + \\
Combretaceae & Combretum micranthum G.Don & + & + \\
Combretaceae & Combretum nigricans Lepr. ex Guill. Et Perr. & & + \\
Combretaceae & Combretum paniculatum Vent. & & + \\
Combretaceae & Guiera senegalensis J.F.Gmel. & + & + \\
Combretaceae & Terminalia macroptera Guill. \& Perott. & + & + \\
\hline & & & +
\end{tabular}




\begin{tabular}{llcc}
\hline Cyperaceae & Cyperus sp. & + & \\
Fabaceae & Pterocarpus erinaceus Poir. & + & + \\
Loranthaceae & Tapinanthus bangwensis (Engl. \& Kr.) Dans. & & + \\
Mimosaceae & Acacia sieberiana DC. & + & + \\
Mimosaceae & Acacia machrostachya Reichenb. Ex DC. & & + \\
Mimosaceae & Acacia seyal Del. & + & + \\
Mimosaceae & Dichrostachys cinerea (L.) Wight \& Arn. & + & + \\
Mimosaceae & Mimosa pigra L. & + & + \\
Mimosaceae & Parkia biglobosa (Jacq.) R.Br.ex G.Don f. & & + \\
Poaceae & Andropogon gayanus Kunth. & + & + \\
Poaceae & Vetiveria nigricana & + & + \\
Rhamnaceae & Ziziphus mucronata Willd. & + & + \\
Rubiaceae & Gardenia ternifolia Schum. \& Thonn. & & + \\
Rubiaceae & Mitragyna inermis (Willd.) O. Ktze. & + & + \\
Rubiaceae & Sarcocephalus latifolius (Smith) Bruce & + & + \\
Sphenocleaceae & Sphenoclea zeylanica & + & + \\
Sterculiaceae & Sterculia setigera Del. & & + \\
Verbenaceae & Stachytarpheta angustifoila & & + \\
\hline$+:$ Présent & &
\end{tabular}

Tableau 6 : Structure de la flore des mares de Nianaka et Dalafourounté.

\begin{tabular}{lcccccccccccc}
\hline & \multicolumn{4}{c}{ Flore de Nianaka } & \multicolumn{4}{c}{ Flore de Dalafourounté } \\
\hline & Familles & Genres & Espèces & Familles & Genres & \multicolumn{1}{c}{ Espèces } \\
\hline & $\mathrm{N}$ & $\%$ & $\mathrm{~N}$ & $\%$ & $\mathrm{~N}$ & $\%$ & $\mathrm{~N}$ & $\%$ & $\mathrm{~N}$ & $\%$ & $\mathrm{~N}$ & $\%$ \\
$\mathrm{D}$ & 10 & 76,9 & 16 & 80 & 20 & 83,3 & 14 & 87,5 & 23 & 88,5 & 32 & 91,4 \\
M & 3 & 23,1 & 4 & 20 & 4 & 16,7 & 2 & 12,5 & 3 & 11,5 & 3 & 8,6 \\
Total & $\mathbf{1 3}$ & $\mathbf{1 0 0}$ & $\mathbf{2 0}$ & $\mathbf{1 0 0}$ & $\mathbf{2 4}$ & $\mathbf{1 0 0}$ & $\mathbf{1 6}$ & $\mathbf{1 0 0}$ & $\mathbf{2 6}$ & $\mathbf{1 0 0}$ & $\mathbf{3 5}$ & $\mathbf{1 0 0}$ \\
\hline
\end{tabular}

$\mathrm{D}$ : Dicotylédone ; $\mathrm{M}$ : Monocotylédone ; $\mathrm{N}$ : nombre d'espèces ; \% : pourcentage.

Tableau 7 : Répartition par famille des espèces des mares de Nianaka et Dalafourounté.

\begin{tabular}{lcccc}
\hline \multirow{2}{*}{ Familles botaniques } & \multicolumn{2}{c}{ Flore de la mare de Nianaka } & \multicolumn{2}{c}{ Flore de la mare de Dalafourounté } \\
\cline { 2 - 5 } & Nombre d'espèces & \% Familles & Nombre d'espèces & \% Familles \\
\hline Anacardiaceae (D) & 1 & 4,2 & 3 & 8,6 \\
Annonaceae (D) & 1 & 4,2 & 1 & 2,9 \\
Apocynaceae (D) & 0 & 0 & 1 & 2,9 \\
Arecaceae (M) & 1 & 4,2 & 1 & 2,9 \\
Bombacaceae (D) & 0 & 0 & 1 & 2,9 \\
Borraginaceae (D) & 1 & 4,2 & 1 & 2,9 \\
Caesalpiniaceae (D) & 3 & 12,5 & 3 & 8,6 \\
Combretaceae (D) & 6 & 25 & 9 & 25,7 \\
Cyperaceae (M) & 1 & 4,2 & 0 & 0 \\
Fabaceae (D) & 1 & 4,2 & 1 & 2,9 \\
& & 2879 & &
\end{tabular}




\begin{tabular}{lcccc}
\hline Loranthaceae (D) & 0 & 0 & 1 & 2,9 \\
Mimosaceae (D) & 4 & 16,7 & 5 & 14,3 \\
Poaceae (M) & 2 & 8,3 & 2 & 5,7 \\
Rhamnaceae (D) & 1 & 4,2 & 1 & 2,9 \\
Rubiaceae (D) & 1 & 4,2 & 3 & 8,6 \\
Sphenocleaceae (D) & 1 & 4,2 & 0 & 0 \\
Sterculiaceae (D) & 0 & 0 & 1 & 2,9 \\
Verbenaceae (D) & 0 & 0 & 1 & 2,9 \\
Total & $\mathbf{2 4}$ & $\mathbf{1 0 0}$ & $\mathbf{3 5}$ & $\mathbf{1 0 0}$ \\
\hline \multicolumn{1}{c}{$\%$ : pourcentage } & & & &
\end{tabular}

Tableau 8 : Indices de fréquences et des pourcentages des espèces dans les mares de Nianaka et Dalafourounté.

\begin{tabular}{|c|c|c|c|c|c|c|c|c|c|c|c|c|}
\hline \multirow{3}{*}{$\begin{array}{l}\text { Indices de } \\
\text { fréquence }\end{array}$} & \multicolumn{4}{|c|}{ Autour de la mare } & \multicolumn{4}{|c|}{ En bordure de la mare } & \multicolumn{4}{|c|}{ A l'intérieur de la mare } \\
\hline & \multicolumn{2}{|c|}{ Nianaka } & \multicolumn{2}{|c|}{ Dalafourounté } & \multicolumn{2}{|c|}{ Nianaka } & \multicolumn{2}{|c|}{ Dalafourounté } & \multicolumn{2}{|c|}{ Nianaka } & \multicolumn{2}{|c|}{ Dalafourounté } \\
\hline & $\mathrm{NE}$ & $\%$ & $\mathrm{NE}$ & $\%$ & $\mathrm{NE}$ & $\%$ & $\mathrm{NE}$ & $\%$ & $\mathrm{NE}$ & $\%$ & $\mathrm{NE}$ & $\%$ \\
\hline $\mathrm{V}$ & 6 & 30 & 9 & 27,3 & 3 & 75 & 2 & 66,7 & 3 & 60 & 1 & 25 \\
\hline IV & 0 & 0 & 1 & 3 & 0 & 0 & 0 & 0 & 2 & 40 & 1 & 25 \\
\hline III & 2 & 10 & 4 & 12,1 & 0 & 0 & 0 & 0 & 0 & 0 & 0 & 0 \\
\hline II & 5 & 25 & 6 & 18,2 & 0 & 0 & 1 & 33,3 & 0 & 0 & 2 & 50 \\
\hline I & 7 & 35 & 13 & 39,4 & 1 & 25 & 0 & 0 & 0 & 0 & 0 & 0 \\
\hline Total & 20 & 100 & 33 & 100 & 4 & 100 & 3 & 100 & 5 & 100 & 4 & 100 \\
\hline
\end{tabular}

Tableau 9 : Répartition des espèces selon les index de Caratini (1985) autour des mares de Nianaka et Dalafourounté.

\begin{tabular}{lll}
\hline Indice & \multicolumn{1}{c}{ Nianaka } & \multicolumn{1}{c}{ Dalafourounté } \\
\hline & Acacia sieberiana & Acacia sieberiana \\
& Combretum glutinosum & Borassus aethiopum \\
& Guiera senegalensis & Cassia sieberiana \\
& Mitragyna inermis & Combretum glutinosum \\
$\mathbf{V}$ & Piliostigma thonningii & Combretum nigricans \\
& Pterocarpus erinaceus & Guiera senegalensis \\
& & Mitragyna inermis \\
& & Piliostigma thonningii \\
& & Pterocarpus erinaceus \\
\hline Total & $\mathbf{6}$ & $\mathbf{9}$ \\
\hline IV & & Terminalia macroptera \\
\hline Total & $\mathbf{0}$ & $\mathbf{1}$ \\
\hline
\end{tabular}




\begin{tabular}{|c|c|c|}
\hline III & $\begin{array}{l}\text { Borassus aethiopum } \\
\text { Mimosa pigra }\end{array}$ & $\begin{array}{l}\text { Acacia seyal } \\
\text { Andropogon gayanus } \\
\text { Hexalobus monopetalus } \\
\text { Lannea microcarpa }\end{array}$ \\
\hline Total & 2 & 4 \\
\hline II & $\begin{array}{l}\text { Anogeissus leiocarpus } \\
\text { Combretum adenogonium } \\
\text { Hexalabus monopetalus } \\
\text { Terminalia macroptera } \\
\text { Ziziphus mucronata }\end{array}$ & $\begin{array}{l}\text { Anogeissus leiocarpus } \\
\text { Bombax costatum } \\
\text { Dichrostachys cinerea } \\
\text { Gardenia ternifolia } \\
\text { Vetiveria nigricana } \\
\text { Ziziphus mucronata } \\
\end{array}$ \\
\hline Total & 5 & 6 \\
\hline I & $\begin{array}{l}\text { Acacia seyal } \\
\text { Andropogon gayanus } \\
\text { Cassia occidentalis } \\
\text { Cassia sieberiana } \\
\text { Combretum micranthum } \\
\text { Dichrostachys cinerea } \\
\text { Lannea microcarpa }\end{array}$ & $\begin{array}{l}\text { Acacia macrostachya } \\
\text { Combretum lecardii } \\
\text { Combretum adenogonium } \\
\text { Combretum micranthum } \\
\text { Combretum paniculatum } \\
\text { Cordyla pinnata } \\
\text { Lannea velutina } \\
\text { Lannea acida } \\
\text { Sarcocephalus latifolius } \\
\text { Parkia biglobosa } \\
\text { Sterculia setigera } \\
\text { Tapinanthus bangwensis } \\
\text { Baissea multiflora }\end{array}$ \\
\hline Total & 7 & 13 \\
\hline
\end{tabular}

Tableau 10 : Répartition des espèces selon les index de Caratini (1985) en bordure et à l'intérieur des mares de Nianaka et Dalafourounté.

\begin{tabular}{|c|c|c|c|c|}
\hline \multirow{2}{*}{ Index } & \multicolumn{2}{|c|}{ Nianaka } & \multicolumn{2}{|c|}{ Dalafourounté } \\
\hline & Bordure & Intérieur & Bordure & Intérieur \\
\hline V & $\begin{array}{l}\text { Mimosa pigra } \\
\text { Mitragyna inermis } \\
\text { Vetiveria nigricana }\end{array}$ & $\begin{array}{l}\text { Mimosa pigra } \\
\text { Cyperus sp. } \\
\text { Coledenia procumbens }\end{array}$ & $\begin{array}{l}\text { Mitragyna inermis } \\
\text { Vetiveria nigricana }\end{array}$ & Vetiveria nigricana \\
\hline Total & 3 & 3 & 2 & 1 \\
\hline IV & & $\begin{array}{l}\text { Sphenoclea zeylanica } \\
\text { Andropogon gayanus }\end{array}$ & & Mitragyna inermis \\
\hline Total & 0 & 2 & 0 & 1 \\
\hline III & & & & \\
\hline Total & 0 & 0 & 0 & 0 \\
\hline II & & & Borassus aethiopum & $\begin{array}{l}\text { Stachytarpheta angustifolia } \\
\text { Coledenia procumbens }\end{array}$ \\
\hline Total & 0 & 0 & 1 & 2 \\
\hline $\mathrm{I}$ & Borassus aethiopum & & & \\
\hline Total & 1 & 0 & 0 & 0 \\
\hline
\end{tabular}


Tableau 11 : Analyse des abondances dominances moyennes, des fréquences et des index (selon Caratini 1985) des espèces recensées autour des mares de Dalafourounté et Nianaka.

\begin{tabular}{|c|c|c|c|c|c|c|}
\hline \multirow{2}{*}{ Espèces } & \multicolumn{3}{|c|}{ Dalafourounté } & \multicolumn{3}{|c|}{ Nianaka } \\
\hline & AD moy & Fréquence & Index & AD moy & Fréquence & Index \\
\hline Acacia macrostachya & 0,02 & 0,2 & I & - & - & - \\
\hline Acacia seyal & 0,7 & 0,6 & III & 0,4 & 0,2 & I \\
\hline Acacia sieberiana & 0,5 & 1 & $\mathrm{~V}$ & 0,6 & 1 & $\mathrm{~V}$ \\
\hline Andropogon gayanus & 0,6 & 0,6 & III & 0,6 & 0,2 & I \\
\hline Anogeissus leiocarpus & 0,5 & 0,4 & II & 0,2 & 0,4 & II \\
\hline Baissea multiflora & 0,02 & 0,2 & I & - & - & - \\
\hline Bombax costatum & 0,2 & 0,4 & II & - & - & - \\
\hline Borassus aethiopum & 0,5 & 1 & $\mathrm{~V}$ & 0,7 & 0,6 & III \\
\hline Cassia occidentalis & - & - & - & 0,02 & 0,2 & I \\
\hline Cassia sieberiana & 0,5 & 1 & $\mathrm{~V}$ & 0,1 & 0,2 & I \\
\hline Combretum adenogonium & 0,02 & 0,2 & I & 0,04 & 0,4 & II \\
\hline Combretum glutinosum & 2 & 1 & $\mathrm{~V}$ & 1,9 & 1 & $\mathrm{~V}$ \\
\hline Combretum lecardii & 0,04 & 0,2 & I & - & - & - \\
\hline Combretum micranthum & 0,1 & 0,2 & I & 0,1 & 0,2 & I \\
\hline Combretum nigricans & 1,5 & 1 & V & - & - & - \\
\hline Combretum paniculatum & 0,02 & 0,2 & I & - & - & - \\
\hline Cordyla pinnata & 0,1 & 0,2 & $\mathrm{I}$ & - & - & - \\
\hline Dichrostachys cinerea & 0,2 & 0,4 & II & 0,1 & 0,2 & I \\
\hline Gardenia ternifolia & 0,2 & 0,4 & II & - & - & - \\
\hline Guiera senegalensis & 1,3 & 1 & V & 3,2 & 1 & $\mathrm{~V}$ \\
\hline Hexalobus monopetalus & 0,3 & 0,6 & III & 0,2 & 0,4 & II \\
\hline Lannea acida & 0,1 & 0,2 & I & - & - & - \\
\hline Lannea microcarpa & 0,7 & 0,6 & III & 0,2 & 0,2 & I \\
\hline Lannea velutina & 0,1 & 0,2 & I & - & - & - \\
\hline Mimosa pigra & - & - & - & 0,3 & 0,6 & III \\
\hline Mitragyna inermis & 1,1 & 1 & $\mathrm{~V}$ & 2,3 & 1 & $\mathrm{~V}$ \\
\hline Sarcocephalus latifolius & 0,1 & 0,2 & I & - & - & - \\
\hline Parkia biglibosa & 0,1 & 0,2 & I & - & - & - \\
\hline Piliostigma thonningii & 1,6 & 1 & $\mathrm{~V}$ & 0,8 & 1 & V \\
\hline Pterocarpus erinaceus & 2,7 & 1 & V & 1,1 & 1 & $\mathrm{~V}$ \\
\hline Sterculia setigera & 0,1 & 0,2 & I & - & - & - \\
\hline Tapinanthus bangwensis & 0,02 & 0,2 & I & - & - & - \\
\hline Terminalia macroptera & 0,9 & 0,8 & IV & 0,2 & 0,4 & II \\
\hline Vetiveria nigricana & 0,2 & 0,4 & II & - & - & - \\
\hline Ziziphus mисronata & 0,2 & 0,4 & II & 0,3 & 0,4 & II \\
\hline
\end{tabular}


Tableau 12 : Analyse des abondances dominances moyennes, des fréquences et des index selon (Caratini 1985) des espèces recensées en bordure des mares de Dalafourounté et Nianaka.

\begin{tabular}{lcccccc}
\hline \multirow{2}{*}{ Espèces } & \multicolumn{3}{c}{ Dalafourounté } & \multicolumn{3}{c}{ Nianaka } \\
\cline { 2 - 7 } & AD moy & Fréquence & Index & AD moy & Fréquence & Index \\
\hline Borassus aethiopum & 0,17 & 0,25 & II & 0,4 & 0,2 & I \\
Mitragyna inermis & 0,83 & 1 & V & 2 & 1 & V \\
Vetiveria nigricana & 5 & 1 & V & 4 & 1 & V \\
Mimosa pigra & - & - & - & 2,6 & 1 & V \\
\hline \multicolumn{1}{c}{ AD moy : Abondances Dominances moyennes }
\end{tabular}

AD moy : Abondances Dominances moyennes

Tableau 13 : Analyse des abondances dominances moyennes, des fréquences et des index selon (Caratini 1985) des espèces recensées à l'intérieur des mares de Dalafourounté et Nianaka.

\begin{tabular}{lcccccc}
\hline \multirow{2}{*}{ Espèces } & \multicolumn{3}{c}{ Dalafourounté } & \multicolumn{3}{c}{ Nianaka } \\
\cline { 2 - 7 } & AD moy & Fréquence & Index & AD moy & Fréquence & Index \\
\hline Andropogon gayanus & - & - & - & 0,33 & 0,67 & IV \\
Coldenia procumbens & 0,17 & 0,33 & II & 0,67 & 1 & V \\
Cyperus sp. & - & - & - & 0,67 & 1 & V \\
Mimosa pigra & - & - & - & 5 & 1 & V \\
Mitragyna inermis & 0,83 & 0,67 & IV & - & - & - \\
Sphenoclea zeylanica & - & - & - & 0,5 & 0,67 & IV \\
Stachytarpheta angustifoila & 0,17 & 0,33 & II & - & - & - \\
Vetiveria nigricana & 3,5 & 1 & V & - & - & - \\
\hline
\end{tabular}

AD moy : Abondances Dominances moyennes

\section{DISCUSSION \\ Caractéristique des sols \\ Granulométrie}

Les sites de Nianaka, Dalafourounté et Kountadala présentent chacun une texture très fine comparativement à Simenti (35 et 50\%) où le sol est argilo-sableux. En effet, pour le cas de Simenti, cette sitation pourrait s'expliquer par le fait que la mare est la plus fréquentée du parc par les visiteurs. Donc, elle est souvent sujette de beaucoup d'activités anthropiques favorisant ainsi l'érosion et le charriage des sédiments et matériaux solides par les eaux de ruissellement des plateaux vers le fond de la mare comme rapporté par Bâ et al. (2008). C'est ce qui fait que la texture soit relativement grossière contrairement aux autres mares. Toutefois, force est de constater qu'il n'y a pas d'effet de granulométrie sur la présence ou l'absence de Mimosa pigra dans ces sites. Dalafourounté en est une illustration parfaite puisque présentant une texture argileuse alors qu'une absence totale de cette espèce a été notée bien que faisant partie du réseau de mares connectées aux cours d'eau du parc. Ce qui s'explique par le fait que ce végétal a une large amplitude écologique qui lui permet de se développer sur des sols à texture différentes comme le rapporte Lonsdale (1988). En effet, cet auteur soutient que Mimosa pigra est trouvée sur une grande variété de sols. De plus, elle ne semble pas se développer de préférence sur un type de sol, mais est le plus souvent trouvée dans les lieux humides tels que les plaines inondables et les berges, sur les sols allant des argiles noires à fissuration en passant aux sols argilo-sableux jusqu'à ceux de type gros sable siliceux le long des rivières. Donc, la granulométrique n'influe pas sur le succès invasif de Mimosa pigra dans le Parc National du Niokolo Koba. 
$p H$

L'ensemble des sites ont enregistré un sol ayant un $\mathrm{pH}$ à l'eau inférieur à 5 alors que le $\mathrm{pH}$ à la Chlorure de potassium affiche des valeurs oscillant entre 3,4 et 3,7. Ces sols sont très acides puisque entrant dans la gamme de $\mathrm{pH}$ compris entre 3,5 et 5 (Baize, 1988). En effet, ce paramètre semble renseigner sur le degré d'infestation des mares par ce végétal ainsi que sur l'envergure de ses populations; Kountadala présente un peuplement monospécifique caractérisé par des fourrés impénétrables composés d'individus feuillus de grande taille. Nianaka, quant à lui, affiche la même situation que le précédent mais avec un niveau de foliation moindre. En ce qui concerne Simenti, il reste une portion d'environ 50\% de la superficie de la mare qui n'est pas encore colonisée par cette espèce (Gueye, 2015). En conclusion, le $\mathrm{pH}_{\mathrm{H} 2 \mathrm{O}}$ est un facteur intervenant dans le succès invasif de Mimosa pigra car le niveau d'infestation des mares étudiées est fonction du degré d'acicité du sol.

\section{Macroéléments}

Les sols des mares de Nianaka, Dalafourounté et Simenti sont riches en azote avec un taux supérieur à $0,125 \%$ par opposition à Kountadala qui est moyennement riche avec une proportion inférieure à $0,075 \%$ comme défini par Diouf (2010). L'analyse de ces résultats par rapport à ceux des travaux de Gueye (2015) permet de dire qu'il n'existe pas de corrélation entre le taux d'azote et le niveau d'occupation de Mimosa pigra dans les mares étudiées. C'est le même cas de figure pour le carbone total, le phosphore total et le phosphore assimilable. Donc, les macroéléments du sols n'ont aucun effet sur l'invasion de Mimosa pigra dans les mares du parc.

\section{Base échangeables et Capacité d'échange cationique}

Les résultats obtenus sur bases échangeables ont montré que le site de Nianaka enregistre la valeur la plus grande (19,65 méq\%), suivi de Dalafourounté (16,35 méq\%). Ces résultats s'expliquent par le fait que le sol des sites de Nianaka, Dalafourounté et Kountadala soient de type argileux lourd alors que celui de Simenti présente une texture argileuse. En définitif, il semble ne pas avoir une corrélation directe entre la capacité d'échange cationique et la présence de Mimosa pigra au niveau des mares étudiées.

\section{Rapport entre éléments minéraux}

Les résultats des rapports entre les macroéléments sont en conformité avec les normes définies par Anonyme (2001) pour tous les sites confondus. En effet, les rapports $\mathrm{C} / \mathrm{N}$ donnent des valeurs comprises entre 8 et 15 , ce qui traduit une alimentation équilibrée en carbone et azote totaux pour les populations de Mimosa pigra. Parallèlement, les rapports $\mathrm{Ca} / \mathrm{K}$ et $\mathrm{Mg} / \mathrm{K}$ variant tous respectivement entre 2 et 17 et entre 2 et 20 s'expliquent par une nutrition minérale favorable en calcium et potassium d'une part et en magnésium et potassium d'autre part (Anonyme, 2001). Par ailleurs, les rapports $(\mathrm{Ca}+\mathrm{Mg}) / \mathrm{K}$ variant entre 2 et 30 traduisent aussi une alimentation favorable en calcium, magnésium et potassium alors que les rapports $\mathrm{Ca} / \mathrm{Mg}$ dont les valeurs sont comprises entre 2 et 4 mettent en évidence une bonne alimentation en ces deux minéraux (Anonyme, 2001). En conclusion, les populations de Mimosa pigra se developpent sur des sols à nutrition minérale correcte.

\section{Etude de la flore \\ Analyse qualitative de la flore}

Comparée à la flore autour de la mare de Nianaka, celle de Dalafourounté présente un nombre de familles, de genres et d'espèces plus élevé et vice versa pour l'intérieur et la bordure des mares. En somme, 35 espèces appartenant à 26 genres répartis en 16 familles ont été recensées à Dalafourounté contre 24 espèces (dont Mimosa pigra) appartenant à 20 genres répartis dans 13 familles à Nianaka. Cette situation serait due non seulement à la présence exclusive de Mimosa pigra à Nianaka, mais également à sa capacité à concurrencer les végétaux comme le rapporte Praneetvatakul (2001). En effet, selon cet 
auteur, cette espèce invasive est une compétitrice féroce de la végétation basse et quelques uns des arbustes capables de réussir en population dense, et sur les pelouses d'herbes hautes. Mimosa pigra peut interférer avec la culture d'autres plantes économiquement importantes. Elle est en mesure de rivaliser avec les jeunes palmiers immatures dans les plantations (Praneetvatakul, 2001). Toutefois, malgré qu'elle soit connue pour être une compétitrice féroce, elle ne peut pas concurrencer avec des plantes qui ont une croissance rapide et un système racinaire bien établi (Janzen, 1983). Ces résultats, comparés à ceux obtenus aux mares de Simenti et Kountadala par Bâ et al. (2008) mettent en évidence l'effet des feux, de l'humidité et du niveau d'infestation par Mimosa pigra sur la présence d'autres espèces végétales au niveau des mares. En effet, selon ces auteurs 58 espèces, 39 et 27 espèces ont été répertoriées respectivement autour, à l'intérieur et en bordure de la mare de Simenti contre 23, 9 et 18 espèces à Kountadala. Parallèlement, chez la première mare la superficie infestée est de $50,94 \%$ comparativement à la seconde qui affiche $99 \%$ et Nianaka $93 \%$ d'après Gueye (2015). Comparativement, la proportion de Dicotylédones $(83,3 \%)$ de Nianaka est inférieure à celle de Dalafourounté $(91,4 \%)$ et vice versa en ce qui concerne les Monocotylédones 16,7\% contre 8,6\%.

Il ressort également de l'analyse que la flore de Nianaka et Dalafourounté présente une dominance de deux familles les Combretaceae et les Mimosaceae. L'importance de la strate arbustive (Combretaceae, Mimosaceae) montre, selon Vanden (1998), une savanisation progressive de la végétation. Ceci est certainement dû à l'usage des feux précoces autour de ces mares au cours des opérations d'aménagement du parc. Au niveau de la mare de Dalafourounté, les résultats obtenus montrent que la famille des Combretaceae regroupe le quart des espèces recensées avec 25,7\%. Cela est dû probablement aux effets conjugués des facteurs climatiques et anthropiques qui se traduisent par la disparition des arbres et la formation d'un tapis discontinu d'espèces herbacées et arbustives comme le rapporte Bassène (2008).

Il apparaît donc que Mimosa pigra est une espèce invasive très compétitive par rapport aux arbustes et herbacées en bordure et à l'intérieur des mares.

\section{Analyse floristique quantitative \\ Etendue de la distribution des espèces dans les mares étudiées}

A Nianaka, à l'intérieur de la mare comme en bordure, Mimosa pigra y est classée parmi les espèces constantes alors qu'elle est fréquente tout autour de la mare où elle est localisée dans les chenaux d'alimentation en eau. Ceci permet de dire que le taux d'infestation de Mimosa pigra est très élevé à l'intérieur et en bordure de Nianaka et que l'infestation vient d'un cours d'eau; ce qui est en conformité avec les travaux de Gueye (2015) qui révèlent un taux d'infestation de $93 \%$. De plus, lorsque la crue du fleuve Gambie atteint une certaine hauteur, les eaux, par le biais des chenaux, inondent les mares les plus importantes telle que Nianaka (Boureima, 2008). Dalafourounté, quant à elle, est caractérisée par la présence constante de Vetiveria nigricana à l'intérieur de la mare et par la même espèce et Mitragyna inermis en bordure. Vetiveria nigricana, espèce fixatrice de berges, couvre une bonne partie de la mare, de la bordure à l'intérieur de celleci. Ce site est aussi marqué par l'absence de Mimosa pigra bien qu'étant alimenté par le fleuve Gambie, principale source d'infestation connue pour le parc. La présence et le taux de recouvrement élevé de Vetiveria nigricana à l'intérieur de la mare de Dalafourounté, pourrait s'expliquer par le fait que la mare est relativement plate, due à l'ensablement et au comblement. Cette topographie ne favorise pas le développement de Mimosa pigra puisque selon Bâ et al. (2008) cette espèce n'est présente que dans les vallées et les 
dépressions inondées pendant une bonne partie de l'année.

Donc, Mimosa pigra est présente exclusivement à la mare de Nianaka, notamment à l'intérieur et en bordure, avec un taux de recouvrement très élevé.

Abondance des espèces

Dalafourounté : Autour de la mare, Pterocarpus erinaceus est l'espèce classée constante qui enregistre l'indice d'abondance dominance moyenne la plus élevée $(2,7)$. Toutes les autres espèces présentent un indice d'abondance dominance moyenne compris entre 0,02 et 2 . Ce qui veut dire que Pterocarpus erinaceus a un recouvrement minimal de $5 \%$ et un maximal de $25 \%$ de la surface autour de la mare. Vetiveria nigricana est la seule espèce constante aussi bien en bordure qu'à l'intérieur de la mare et présentant une abondance dominance moyenne élevée de 5 et 3,5 respectivement. Ceci s'explique par le fait que cette espèce a un taux de recouvrement compris entre 75 et $100 \%$ de la surface en bordure et de 25 à $50 \%$ de la surface de l'intérieur de la mare comme définit par Gillet (2000).

Nianaka: Guiera senegalensis a une abondance dominance moyenne élevée et égale à 3,2 correspondant à un taux de recouvrement compris entre 25 et $50 \%$ de la surface autour de la mare selon Gillet (2000). Par la même occasion, Mimosa pigra couvre entre 0 et $0,1 \%$ de cette surface. En bordure de la mare, Vetiveria nigricana, Mimosa pigra et Mitragyna inermis sont constantes et montrent une abondance dominance moyenne élevée et égale à 4, 2,6 et 2 respectivement. Ce qui se traduit par un recouvrement de 50 à $75 \%$ pour Vetiveria nigricana et 5 à $25 \%$ pour les deux autres (Gillet, 2000). Dans la mare, Mimosa pigra est la seule espèce constante présentant une abondance dominance moyenne élevée de 5. Ce résultat se traduit par un taux de recouvrement de cette espèce à l'intérieur de la mare compris entre 75 et $100 \%$ d'après Gillet (2000). Ce qui est en concordance avec les résultats des travaux de Gueye (2015) qui révèlent que le taux d'occupation de cette espèce dans la mare de Nianaka est $93 \%$. Donc, Mimosa pigra est présente au niveau de la mare de Nianaka avec un degré d'infestation très élevé.

\section{Conclusion}

Dans cette étude, nous avons voulu, dans un contexte d'invasion biologique par Mimosa pigra, caractériser aux plans pédologique et phytosociologique, les mares de Nianaka et de Dalafourounté, toutes deux localisées dans le Parc National du Niokolo Koba. Les résultats obtenus montrent que ces sites présentent chacun une texture très fine et une absence d'effet de la granulométrie sur présence ou l'absence de Mimosa pigra dans les mares. De plus, le $\mathrm{pH}_{\mathrm{H} 2 \mathrm{O}}$ est un facteur intervenant dans le succès invasif de cette espèce dans ces mares caractérisées par richesse en azote. Au plan phytosociologique, il apparaît que Mimosa pigra est présente exclusivement à la mare de Nianaka avec un taux d'occupation très élevé, notamment à l'intérieur et en bordure de celle-ci. En outre, Mimosa pigra s'est révélée très compétitive par rapport à la végétation arbustive et herbacée en bordure et à l'intérieur des mares. Cette étude devrait être étendue sur toutes les mares du parc, envahies ou non par Mimosa pigra et susceptibles de l'être, afin de mieux cerner cette problématique d'invasion biologique. Des recherches sur le comportement des populations de cette invasive par rapport au $\mathrm{pH}_{\mathrm{H} 2 \mathrm{O}}$ devraient être approfondies.

\section{CONFLIT D'INTERET}

Les auteurs déclarent qu'il n'y a aucun conflit d'intérêt pour cet article.

\section{CONTRIBUTIONS DES AUTEURS}

La collecte de données de terrain, leur traitement, leur analyse et la rédaction du manuscrit ont été assurés par MG alors que KN s'est chargé de la relecture et de la mise au point de l'article. Tous les auteurs ont participé à l'élaboration du manuscrit et ont donné leur accord pour sa publication. 


\section{REMERCIEMENTS}

Mes remerciements vont à l'endroit de Monsieur César Bassène enseignant-chercheur à l'Université Gaston Berger de Saint-Louis et à Monsieur Camara, responsable de l'herbier du département de biologie végétale à l'Université Cheikh Anta Diop de Dakar de m'avoir aider à l'identification de certaines espèces végétales. J'exprime toute ma gratitude à Monsieur Dominique Masse et à Madame Cormier de l'Institut de Recherche pour le Développement (IRD) pour leur appui combien appréciable aux analyses pédologiques.

\section{REFERENCES}

Adam JG. 1971. Le milieu biologique, flore et végétation. In Le Niokolo Koba, Premier Grand Parc National de la République du Sénégal. G.I.A : Dakar ; 43-62.

Anonyme. 2001. Cours de pédologie appliquée II. Ecole Nationale Supérieure d'Agriculture (ENSA) de Thiès.

Arbonnier M. 2010. Arbres, Arbustes et Lianes des Zones Sèches d'Afrique de l'Ouest. CIRAD/MNHN, 576 p.

Bâ AT, Noba K, Sambou B, Goudiaby A, Mbaye MS. 2008. Etude botanique et éco-physiologique de Mimosa pigra et Mitragyna inermis pour une stratégie de contrôle de ces plantes envahissantes dans les mares de Simenti et de Kountadala du Parc National du Niokolo Koba (Sénégal). 49p+ annexes.

Bassène C. 2008. Hyptis suaveolens (1.) Poit. (Lamiaceae) dans les systèmes agropastoraux de la communauté rurale de Mlomp : étude de quelques aspects de la biologie, de l'écologie et proposition de méthodes de contrôle. Mémoire de DEA, Université Cheikh Anta Diop de Dakar, Dakar. 107 p.

Bérhaut J. 1967. Flore du Sénégal, deuxième édition, Clairafrique, Dakar, 485p.

Bérhaut J. 2011. Nouvelle Flore illustrée du Sénégal et des régions voisines. Version électronique, édition complète réalisée par Jacques MUGNIER
Biaze D. 1988. Guide des analyses courantes en pédologie. Institut National de la recherche agronomique, $172 \mathrm{p}$.

Boureima A. 2008. Réserves de biosphère en Afrique de l'Ouest, 68p.

Braun-Blanquet J. 1932. Plant Sociology. The Study of Plant Communities. New York, London: McGray Hill; 439p.

Caratini R, 1985. Botanique 4. Bordas : Paris, France.

Cronk QB, Fuller JL. 1995. Plant Invaders: The Threat to Natural Ecosystems. Chapman \& Hall : London, UK.

Diouf F. 2010. Détermination du potentiel infectieux en rhizobiums symbiotiques de Mimosa pigra et Mimosa pudica de sols du Sénégal. Mémoire de DEA, 59p tannexes.

DPN. 2000. Plan de gestion du Parc National du Niokolo Koba et de sa périphérie. Direction des Parcs Nationaux. Ministère de l'Environnement.131p.

Gillet F. 2000. La phytosociologie synusiale intégrée: Guide méthodologique, Université de Nauchâtel-Institut de Botanique. Docu. Labo. Ecol. Vég. 68 p.

Godron M. 1968. Quelques applications de la notion de fréquence en Ecologie végétale. Oecol. Plant, 3 : 185 - 212

Gueye M. 2014. Modélisation climatique et pédologique de la distribution de Mimosa pigra L.: Analyse de sa dynamique invasive en Afrique et au Parc National du Niokolo Koba. Travail de Fin d'Etudes, Université de Liège, Belgique. $69 p$.

Gueye M. 2015. Dynamique invasive de Mimosa pigra dans un contexte de variabilité du régime hydrologique au Sénégal. Journal of Animal \& Plant Sciences, 26(1): 4023-4035.

Jamagne M. 1967. Base et techniques d'une cartographie des sols. Ann. Agron., 18 : $142 \mathrm{p}$.

Lonsdale WM. 1988. Litterfall in an Australian population of Mimosa pigra, an invasive tropical shrub. Journal of Tropical Ecology, 4: 381-392. 
Lonsdale WM, Miller IL, Forno IW. 1989. The Biology of Australian Weeds 20. Mimosa pigra L. Plant Protection Quarterly, 4(3): 119 - 130.

Lonsdale WM, Miller IL, Forno IW. 1995. Mimosa pigra L. In The biology of Australian Weeds, Groves RH, Sheppard RCH, Richardson RG (eds). R.G. and F.J. Richardson Publishers: Melbourne, Australia; 169-188

Lowe S, Browne M, Boudjelas S, De Poorter M. 2000. 100 of the World's Worst Invasive Alien Species A selection from the Global Invasive Species Database. Published by The Invasive Species Specialist Group (ISSG) a specialist group of the Species Survival Commission (SSC) of the World Conservation Union (IUCN), 12pp. First published as special lift-out in Aliens 12, December 2000. Updated and reprinted version: November 2004.

McNeely AJ, Mooney HA, Neville LE, Schei PJ et Waage JK. 2001. Global Invasive Species Programme

Praneetvatakul S. 2001. An Impact Assessment of ACIAR Research Projects on Biological Control in Thailand. In Impact Assessment of Forty-Nine Thailand/Australia Collaborative Projects Funded by ACIAR during 1983 - 1995 (Working Paper Series No.38), S
Isvilanonda, $\mathrm{S}$ Praneetvatakul, $\mathrm{C}$ Sangkapituk, A Sattarasart, C Singhaprecha, P Sirisupluxana.

Ndiaye A. 2000. Etat des lieux des principales mares et salines du Niokolo Koba. Projet FAC/FEM de Réhabilitation du Parc National du Niokolo Koba et de sa périphérie.

Ndiaye M. 2012. Évaluation de l'efficacité de la gestion du Parc National de Niokolo Koba (Sénégal). Travail de Fin d'Études, Université de Liège, Belgique. 106p.

Renaud PC, Gueye MB, Hejcmanová P, Antoninova M, Samb M. 2006. Inventaire aérien et terrestre de la faune et relevé des pressions au Parc National du Niokolo Koba, 44p.

Schneider A, Sambou K. 1982. Prospection botanique dans les Parc nationaux du Niokolo Koba et de Basse Casamance : Recherche scientifique dans les parcs nationaux du Sénégal, Mémoire de l'IFAN, n92, pp.101-102.

Vanden BC. 1982. Initiation à l'étude de la végétation. $3^{\mathrm{e}}$ Edn, Jardin Botanique National de Belgique, 263 p.

Walden D, Finlayson CM, Van Dam R, Storrs M. 1999. Information for a risk assessment and management of Mimosa pigra in Tram Chim National Park, Viet Nam. Proceeding of the EnviroTox'99 International Conference, 160 - 170. 